\title{
О МАССЕ И СИЛЕ
}

\section{Введение}

Масса и сила относятся к числу основных понятий механики, притом не только классической, но и релятивистской. Между тем обоснование этих понятий в существующей учебно-методической и научной литературе оставляет желать лучшего. Уже давно были осознаны недостатки первоначального, идущего от Ньютона, способа введения этих величин. Предпринимались различные попытки улучшения системы основных понятий и аксиом механики. Г. Герц счел, например, целесообразным обойтись при построении механики вообще без понятия силы [ $\left.{ }^{1}\right]$. Однако ни эта, ни многие другие попытки решающего успеха не имели. Теория относительности (специальная) принесла с собой новые возможности, но они по разным причинам остались в полной мере неиспользованными. Наоборот, вместо ясности возникли новые недоразумения. Так, до сих пор нет еще в литературе твердого мнения о массе: то массой является только масса покоя, то полная масса; то свет имеет массу, то нет и т. д.

Более или менее общепризнано, что второй и третий законы Ньютона не являются только законами, но включают в себя полностью или частично определение силы и массы. Общим признанием пользуется также принцип операциональности определений. Однако конкретное осуществление этих бесспорных положений далеко не общепринято, причем нередко имеет место непоследовательность или неэффективность. Не углубляясь в подробный анализ всех представленных в литературе взглядов, поставим себе целью изложение нового способа обоснования понятий массы и силы, а также других, связанных с ними основных понятий механики. Этот способ, как нам кажется, свободен от традиционных недостатков и обладает рядом существенных преимуществ.

\section{Основные предпосылки}

В основу нашего метода мы положим две руководящие мысли. Первая заключается в максимально возможном сближении классической и релятивистской механики. Это значит, что способ введения основных понятий должен быть в обеих механиках один и тот же. Одинаковы должны быть определения величин и основные закономерности. Для этой цели мы должны отобрать из той и другой механики то, что в их основах является общим. Различие должно проявиться лишь во вторую очередь, вне сферы общих принципов. Это требование вытекает, во-первых, из возросшей в наше время роли релятивистской механики, которая уже 
давно утратила характер чисто теоретического построения и нашла важные технические применения. Не случайно основы теории относительности введены в программу средней школы. Но нет большого смысла обучать классической и релятивистской механике раздельно. Объединение основ обеих механик даст, помимо выигрыша времени, более глубокое проникновение в сущность. Во-вторых, необходимость «релятивизации» основ классической механики диктуется тем малозамеченным до сих пор обстоятельством, что механика Ньютона в своих основах не вступает в противоречие с постулатами теории относительности (см. по этому поводу $\left.\left[{ }^{2}\right]\right)$.

Другая руководящая мысль состоит в том, что метод должен опираться на законы сохранения, в первую очередь на закон сохранения импульса. Это требование является в сущности развитием нашей первой руководящей мысли, поскольку закон сохранения импульса является общим для обеих механик. В традиционном изложении классической механики этот закон рассматривается как следствие второго и третьего законов Ньютона. Однако, как хорошо известно, третий закон Ньютона имеет ограниченную применимость. Он соответствует концепции дальнодействия и теряет силу в теории поля. В теории относительности, основанной на концепции близкодействия, третий закон Ньютона по меньшей мере ненужен. Но закон сохранения импульса остается тем не менее в силе всегда, независимо от применимости или неприменимости третьего закона Ньютона. Будучи, следовательно, более общим по сравнению с последним, закон сохранения импульса скорее, чем тот, годится в качестве основного закона механики. Вдобавок следует подчеркнуть фундаментальный характер законов сохранения вообще. Если во время Ньютона об этой фундаментальной роли было мало известно, то в наше время ее трудно переоценить.

\section{Об операциональном определении массы}

Требование операциональности определений означает, что определение какой-либо величины должно включать указание способа ее измерения. Но первым и непременным условием возможности измерения является возможность установления равенства. Отсюда следует, что первичное операциональное определение массы, по-видимому, вообще невозможно. В самом деле, нельзя придумать никакого метода, который позволил бы, не обращаясь к другим величинам, обнаружить равенство масс двух тел. Но с помощью других величин это возможно. В литературе принято сравнивать массы или по весу (см., напр., $\left.\left[{ }^{3,4}\right]\right)$, или по ускорению, сообщаемому им одинаковыми силами (см., напр., $\left.\left[{ }^{5,6}\right]\right)$. Оба эти способа неудовлетворительны. Первый потому, что он дает тяжелую, но не инертную массу; второй потому, что, не согласуясь с релятивистской механикой, он противоречит нашей первой руководящей мысли. Кроме того, недостатком этого способа является сведение измерения массы к измерению силы, а вопрос об измерении сил далеко не прост. Мы предлагаем взамен операциональное определение массы, основанное на определении и измерении импульса. Об этом ниже.

\section{Два основных закона механики}

Первым основным законом механики является общеизвестный принцип относительности. Второй закон - закон сохранения импульса - включает в себя операциональное определение импульса. Остановимся не- 
сколько подробнее на этом определении и на опытном обосновании самого закона. Рассмотрим полностью неупругие соударения тел (или материальных объектов иного рода, например, электромагнитных волн), т. е. такие соударения, в результате которых оба объекта сливаются в один вторичный объект. Опыт показывает, что если два объекта движутся с определенными, противоположно направленными скоростями, то при их полностью неупругом соударении вторичный объект или остается неподвижным, или движется в направлении движения одного из первичных объектов. В первом случае мы имеем основание охарактеризовать движение объектов некоторой векторной, параллельной скорости величиной и приписать объектам равные и противоположные значения этой величины, дающие в сумме нуль. Эту величину мы и называем импульсом. Подчеркнем, что равенство импульсов устанавливается непосредственным первичным опытом без ссылки на какую-либо другую величину. Решающим обстоятельством является здесь векторный характер импульса. Наоборот, как выше отмечено, для массы подобный непосредственный опыт невозможен, что обусловлено скалярностью этой величины (две массы нельзя, не прибегая к силам, «уравновесить»).

Вернемся к неупругим соударениям. Если вторичный объект не покоится, то его импульс отличен от нуля, а импульсы первичных объектов неравны. Приняв произвольно какой-либо импульс за единицу, можно измерить путем повторных опытов того же типа любой импульс, т. е. найти его кратность относительно единичного импульса. Затем опыт показывает, что измеряемый таким образом импульс сохраняется во всех взаимодействиях объектов - не только при полностью неупругих, но и при частично неупругих и полностью упругих соударениях и вообще в любых процессах. Это и есть второй, после принципа относительности, основной закон механики.

\section{Обоснование понятия массы}

Теперь мы имеем возможность обосновать понятие массы. Так как импульс $p_{k}$ любого объекта, движущегося со скоростью $u_{k}$, параллелен скорости, то можно написать формулу

$$
p_{k}=m u_{k}, \quad k=1,2,3,
$$

где $m-$ некоторый положительный скалярный множитель, называемый массой объекта. Выясним свойства массы.

С этой целью воспользуемся законом сохранения импульса, который должен выполняться, в силу прннципа относительности, в любой инерциальной системе. Пусть пространственные координаты $x_{k}$ и время $t$ преобразуются при переходе в другую инерциальную систему согласно линейным однородным формулам

$$
\begin{aligned}
& x_{k}^{\prime}=\alpha_{k l} x_{l}+\alpha_{k 0} t, \\
& t^{\prime}=\alpha_{0 l} x_{l}+\alpha_{00} t,
\end{aligned}
$$

где коэффициенты $\alpha_{k l}, \alpha_{k 0}, \alpha_{0 l}$ и $\alpha_{00}$ зависят от относительной скорости обеих систем. Линейность и однородность обосновываются, как обычно, однородностью пространства и времени. Вид формул (2) нет нужды конкретизировать; таким образом, наши дальнейшие выводы одинаково верны как в классическом случае преобразований Галилея, так и в релятивистском случае преобразований Лоренца. 
Выражая производные $u_{k}^{\prime}=d x_{k}^{\prime} / d t^{\prime}$ через производные $u_{l}=d x_{l} / d t$, находим

$$
u_{k}^{\prime}=\left(\alpha_{k l} u_{l} f \alpha_{k 0}\right) /\left(\alpha_{0 l} u_{l}+\alpha_{00}\right) .
$$

Импульс выражается в другой инерциальной системе формулой, аналогичной $(1)$ :

$$
p_{k}^{\prime}=m^{\prime} u_{k}^{\prime}
$$

Подставляя сюда вместо $u^{\prime}{ }_{k}$ выражение из формулы (3), с заменой в ней $u_{l}=p_{l} / m$, находим

$$
p_{k}^{\prime}=m^{\prime}\left(\alpha_{k l} p_{l} f \alpha_{k 0} m\right) /\left(\alpha_{0 l} p_{l}+\alpha_{00} m\right) .
$$

Для того, чтобы импульс сохранялся в каждой инерциальной системе, необходимо, чтобы компоненты его преобразовывались линейно. Следовательно, из формулы (5) имеем

$$
\begin{aligned}
& p_{k}^{\prime}=q\left(\alpha_{k l} p_{l}+\alpha_{k 0} m\right), \\
& m^{\prime}=q\left(\alpha_{0} p_{l}+\alpha_{00} m\right),
\end{aligned}
$$

где $q-$ не зависящая от импульса постоянная. Она может зависеть только от относительной скорости обеих инерциальных систем, и притом, в силу изотропности пространства, только от абсолютной величины скорости, но не от ее направления. Убедимся, что $q=1$. В самом деле, введя матрицы

$$
\begin{gathered}
\alpha=\left(\begin{array}{ll}
\alpha_{k l} & \alpha_{k 0} \\
\alpha_{0 l} & \alpha_{00}
\end{array}\right), \\
x=\left(\begin{array}{c}
x_{k} \\
t
\end{array}\right)
\end{gathered}
$$

и

$$
p=\left(\begin{array}{c}
p_{k} \\
m
\end{array}\right)
$$

перепишем формулы (2) и (6) в виде

$$
\begin{aligned}
& x^{\prime}=\alpha x, \\
& p^{\prime}=q \alpha p .
\end{aligned}
$$

Из формулы (10) видно, что $\alpha^{-1}$ есть матрица обратного преобразования; следовательно,

$$
p=q \alpha^{-1} p^{\prime}
$$

Подставляя сюда вместо $p^{\prime}$ выражение из формулы (11), находим $p=q^{2} p$, откуда $q=1$ (значение $q=-1$ исключается, так как оно не согласуется со случаем тождественного преобразования). Итак, окончательно,

$$
\begin{aligned}
& p_{k}^{\prime}=\alpha_{k l} p_{l}+\alpha_{k 0} m, \\
& m^{\prime}=\alpha_{0 l} p_{l}+\alpha_{00} m,
\end{aligned}
$$

или, в матричном виде,

$$
p^{\prime}=\alpha p,
$$


Этот результат означает, что импульс и масса преобразуются в точности так же, как радиус-вектор и время. Другое важное следствие состоит в том, что масса, введенная вначале формально равенством (1), оказывается сохраняющейся во всех инерциальных системах величиной. Это видно из формул (13), если перепишем их для произвольной замкнутой совокупности объектов:

$$
\begin{aligned}
& \sum_{i} p_{k}^{(i)^{\prime}}=\alpha_{k l} \sum_{i} p_{l}^{(i)}+\alpha_{k 0} \sum_{i} m^{(i)}, \\
& \sum_{i} m^{(i)^{\prime}}=\alpha_{0 l} \sum_{i} p_{l}^{(i)}+\alpha_{00} \sum_{i} m^{(i)} .
\end{aligned}
$$

В первой из этих формул левая часть и первый член правой части являются сохраняющимися величинами; следовательно, сохраняется и полная масса совокупности (второй член правой части) в первой инерциальной системе. Согласно второй формуле (15), масса сохраняется и в другой инерциальной системе.

Итак, мы получили обоснование понятия массы как сохраняющейся величины, измерение которой сводится, согласно формуле (1), к измерению скорости и импульса.

\section{Сила}

Располагая понятием импульса как сохраняющейся величины, приходим легко к операциональному определению силы. Так как в совокупности взаимодействующих объектов импульс каждого объекта в отдельности не сохраняется, то естественно ввести меру изменения импульса во времени. Эта мера - скорость изменения импульса и есть сила:

$$
\mathbf{F}=d \mathbf{p} / d t
$$

Данное операциональное определение силы включает в себя, очевидно, второй закон Ньютона в форме, одинаковой и для классической, и для релятивистской механики.

\section{Заключение}

Мы сформулировали основные законы механики - принцип относительности и закон сохранения импульса - и обосновали понятия массы и силы. Этот способ одинаков как для классической, так и для релятивистской механики. Заметим, что в механику у нас включены не только тела, но и любые объекты, обладающие импульсом (см. по этому поводу $\left.\left[{ }^{7}\right]\right)$. Расхождение между классической и релятивистской механиками начинается с введения второго постулата Әйнштейна, прямым следствием которого являются преобразования Лоренца (10) и, в силу формулы (14), неинвариантность массы. В применении к телам, обладающим ненулевой массой покоя, это означает зависимость массы от скорости. Вид зависимости, в силу тех же формул (10) и (14), совпадает с формулой, связывающей длительность процесса во времени произвольной инерциальной системы с длительностью того же процесса в собственном времени. Наоборот, отказ от второго постулата Эйнштейна влечет за собой инвариантность массы и абсолютность времени (c.M. $\left.\left[{ }^{8}\right]\right)$. 


\section{ЛИТЕРАТ У РА}

1. Герц Г., Принципы механики, изложенные в новой связи, М., Изд-во АН СССР, 1959.

2. К а рд П. Г., Вопросы методики преподавания физики в вузе. В помощь преподавателю VI, изд. ТГУ (в печати).

3. Р од же р с Э., Физика для любознательных, т. 1, М., «Мир», 1969, с. 34-35.

4. Эл ли от Л., У и лк ок с У., Физика, М., «Наука», 1967, с. 53-54.

5. М э и о н Дж. Б., Физика и физический мир, М., «Мнр», 1975, с. 141.

6. Иос Г., Курс теоретической физнки, ч. 1, М., Учпедгиз, 1963, с. 86.

7. К а р д П., Изв. АН ЭССР, Физ. Матем., 25, № 1, 15-22 (1976).

8. К а р д П., Изв. АН ЭССР, Физ. Матем., 25, № 3, 227-233 (1976).

\section{Тартуский государственный университет}

\section{Поступила в редакцию} 24/III 1980

\section{P. KARD}

\section{MASS JA JOUUD}

Võttes mehaanika põhiseadusteks impulsi jäävuse seaduse ja relatiivsusprintsiibi ning defineerides mistahes antud objekti massi tema impulsi ja kiiruse jagatisena, leitakse impulsi komponentide ja massi jaoks teisendusvalemid, mis ühtivad kujult aja ja ruumikoordinaatide teisendusvalemitega. Nendest valemitest järeldub otseselt massi jäävus. Jōud defineeritakse üksikobjekti impulsi muutumise kiirusena. Selle meetodi eelisteks on kõikide definitsioonide täielik operatsionaalsus ja relativistliku ning mitterelativistliku mehaanika aluste ühtsus. Oleminek mitterelativistlikult mehaanikalt relativistlikule algab Einsteini teise postulaadi sissetoomisest ja Galilei teisenduste asendamisest Lorentzi teisendustega. See toob otsekohe kaasa massi mitteinvariantsuse, ilma et muutuks selle mõiste põhimõtteline tähendus.

\section{P. KARD}

\section{MASSE UND KRAFT}

Postuliert man als die Grundgesetze der Mechanik das Relativitätsprinzip und die Erhaltung des Impulses, und definiert man die Masse eines Objektes als den Quotienten seines Impulses durch die Geschwindigkeit, so erhält man für die Masse und die Komponenten des Impulses Transformationsformeln, die dieselbe Gestalt haben als die der Zeit und der Raumkoordinaten. Aus diesen Formeln folgt die Erhaltung der Masse. Die Kraft definiert man als die Anderung des Impulses eines einzelnen Objektes je Zeiteinheit. Als Vorzüge dieser Methode sind die völlige Operationalität aller Definitionen und die prinzipielle Einheit der relativistischen und nichtrelativistischen Mechanik zu nennen. Der Ubergang von der nichtrelativistischen Mechanik zu der relativistischen beginnt mit der Einführung des zweiten Postulats Einsteins. Das führt die LorentzTransformationen statt der Galilei-Transformationen an Platz, woraus sofort die Nichtinvarianz der Masse folgt. Jedoch bleibt dabei der prinzipielle Inhalt des Massenbegriffes völlig unverändert. 